\title{
GRADED ASSOCIATIVE CONFORMAL ALGEBRAS OF FINITE TYPE
}

\author{
PAVEL KOLESNIKOV
}

\begin{abstract}
In this paper, we consider graded associative conformal algebras. The class of these objects includes pseudo-algebras over non-cocommutative Hopf algebras of regular functions on some linear algebraic groups. In particular, an associative conformal algebra which is graded by a finite group $\Gamma$ is a pseudo-algebra over the coordinate Hopf algebra of a linear algebraic group $G$ such that the identity component $G^{0}$ is the affine line and $G / G^{0} \simeq \Gamma$. A classification of simple and semisimple graded associative conformal algebras of finite type is obtained.
\end{abstract}

\section{INTRODUCTION}

Conformal algebras were introduced in [1] as a useful tool for studying vertex algebras appeared in two-dimensional conformal field theory in mathematical physics [2. The structure of a (Lie) conformal algebra encodes the singular part of the operator product expansion (OPE) which is responsible for the commutator of two chiral fields.

From the algebraic point of view, the notions of conformal algebras, their representations and cohomologies are higher-level analogues of the ordinary notions in the pseudo-tensor category [3] associated with the polynomial Hopf algebra $\mathbb{k}[D]$, see 4] for details.

For an arbitrary Hopf algebra $H$, an algebra in the pseudo-tensor category associated with $H$ is called pseudo-algebra. Note that ordinary algebras (representations, cohomologies) correspond to the "trivial" case of 1-dimensional Hopf algebra. For pseudo-algebras, the analogues of finite-dimensional algebras are those finitely generated as modules over $H$. These pseudo-algebras are said to be of finite type or simply finite.

The structure theory of finite Lie conformal algebras was developed in [5] and later generalized in 4 for pseudo-algebras over a wide class of cocommutative Hopf algebras. The structure theorems for finite associative conformal algebras and pseudo-algebras over cocommutative Hopf algebras can be derived from the corresponding statements on Lie algebras.

Our aim is to obtain structure theorems for finite associative pseudo-algebras over some non-cocommutative Hopf algebras (Lie pseudo-algebras can not be defined in this case).

In [6], we proposed the notion of a conformal algebra over a linear algebraic group $G$ which is equivalent to the notion of a pseudo-algebra over the Hopf algebra $\mathbb{k}[G]$ of regular functions on $G$. Ordinary algebras over a field $\mathbb{k}$ correspond to the case of trivial group, conformal algebras - to the case when $G$ is isomorphic to the affine line $\mathbb{A}^{1}=(\mathbb{k},+)$. 
In this paper, we introduce the notion of a graded conformal algebra over a (connected) abelian linear algebraic group $G^{0}$. In contrast to the case of ordinary algebras, we need three parameters to define the grading: a group $\Gamma$, a homomorphism $\sigma: \Gamma \rightarrow$ Aut $G^{0}$, and a 2-cocycle $\varphi$ of the group $\Gamma$ with coefficients in $G^{0}$ endowed with $\Gamma$-module structure $\gamma \lambda=\lambda^{\sigma(\gamma)}, \gamma \in \Gamma, \lambda \in G^{0}$. The main reason for this approach is the following: If $|\Gamma|<\infty$ then a $(\Gamma, \sigma, \varphi)$-graded conformal algebra over $G^{0}$ is the same as conformal algebra over a linear algebraic group $G$ obtained as the extension of $G^{0}$ by means of $\Gamma$ with respect to $\sigma$ and $\varphi$. However, the group $\Gamma$ may be infinite. In this case, a $(\Gamma, \sigma, \varphi)$-graded conformal algebra is no more a pseudo-algebra in the sense of [4].

The main result of the paper is the classification of finite simple and semisimple associative $(\Gamma, \sigma, \varphi)$-graded conformal algebras over $\mathbb{A}^{1}$.

\section{Preliminaries in CONFOrmal algebras And PSEUdo-Algebras}

Suppose $A$ is an algebra (not necessarily associative) over a field $\mathbb{k}$, char $\mathbb{k}=0$. Denote by $A\left[\left[z, z^{-1}\right]\right]$ the space of formal power series (distributions) that may be infinite in both directions. A pair of distributions $a(z), b(z) \in A\left[\left[z, z^{-1}\right]\right]$ is called local if there exists a natural $N$ such that $a(w) b(z)(w-z)^{N}=0$ in the space $A\left[\left[z, z^{-1}, w, w^{-1}\right]\right]$. The product $a(w) b(z)$ of two formal distributions that form a local pair may be presented as

$$
a(w) b(z)=\sum_{n=0}^{N-1} \frac{1}{n !} c_{n}(z) \frac{\partial^{n} \delta(w-z)}{\partial z^{n}},
$$

where $\delta(w-z)=\sum_{m \in \mathbb{Z}} w^{m} z^{-m-1}$ is the formal delta-function, $c_{n}(z) \in A\left[\left[z, z^{-1}\right]\right]$ [1].

For example, if $V$ is a vertex operator algebra (see 7] as a general reference) with a state-field correspondence $Y: V \rightarrow \operatorname{gl} V\left[\left[z, z^{-1}\right]\right], Y: a \mapsto Y(a, z)$, then the space of all distributions $\{V(a, z) \mid a \in V\}$ consists of pairwise mutually local series.

For a local pair of distributions $a(z), b(z) \in A\left[\left[z, z^{-1}\right]\right]$ one may consider the Fourier transform of their product

$$
\left(a_{(\lambda)} b\right)(z)=\operatorname{Res}_{w=0} a(w) b(z) \exp \{\lambda(w-z)\},
$$

where $\operatorname{Res}_{w=0} f(w, z, \lambda)$ is the formal residue of $f$, i.e., the coefficient at $w^{-1}$. Locality implies the $\lambda$-product $\left(a_{(\lambda)} b\right)$ to be a polynomial in $\lambda$ with coefficients in $A\left[\left[z, z^{-1}\right]\right]$. Axiomatic description of the properties of this new "parametrized" operation and its relationship with the ordinary derivation $T=d / d z$ on $A\left[\left[z, z^{-1}\right]\right]$ leads to the following

Definition 1 ([1]). A conformal algebra is a linear space $C$ over a field $\mathbb{k}$ of zero characteristic endowed with a linear map $T: C \rightarrow C$ and bilinear operation $(\cdot(\lambda) \cdot)$ : $C \otimes C \rightarrow C[\lambda]$ such that $\left(T a_{(\lambda)} b\right)=-\lambda\left(a_{(\lambda)} b\right)$ and $\left(a_{(\lambda)} T b\right)=(T+\lambda)\left(a_{(\lambda)} b\right)$.

Conformal algebra which is finitely generated as $\mathbb{k}[T]$-module is said to be finite.

A subspace $C \subseteq A\left[\left[z, z^{-1}\right]\right]$ that consists of pairwise mutually local series which is closed under $d / d z$ and $(\cdot(\lambda) \cdot)$ given by (11) is called a conformal algebra of formal distributions over $A$.

For every conformal algebra $C$ there exists a unique (up to isomorphism) coefficient algebra $\mathcal{A}(C)$ [8] such that $C$ is embedded into a conformal algebra of formal 
distributions over $\mathcal{A}(C)$ and $\mathcal{A}(C)$ is universal among all such algebras $A$. The following natural definition was proposed in [8]: Given a variety $\mathfrak{M}$ of algebras (associative, commutative, Lie, etc.), a conformal algebra $C$ is said to be $\mathfrak{M}$-conformal algebra if and only if $\mathcal{A}(C)$ belongs to $\mathfrak{M}$. In particular, every vertex operator algebra gives rise to a Lie conformal algebra. This is the main motivation to study Lie conformal algebras and their representations.

Structure theory of finite Lie and associative conformal algebras was developed in [5]. In particular, if $\mathbb{k}$ is an algebraically closed field of zero characteristic then every simple finite associative conformal algebra $C$ is isomorphic to the current conformal algebra over $A=M_{n}(\mathbb{k})$, i.e.,

$$
C \simeq \mathbb{k}[T] \otimes A, \quad(f(T) \otimes a)_{(\lambda)}(g(T) \otimes b)=f(-\lambda) g(T+\lambda) \otimes a b,
$$

for $f, g \in \mathbb{k}[T], a, b \in A$. Conformal algebra is called semisimple if it has no nonzero ideals $I$ such that $I_{(\lambda)} I=0$. A semisimple finite associative conformal algebra is proved to be a direct sum of simple ones $[5$.

Note that for finite Lie conformal algebras there exists a unique exceptional example apart from current conformal algebras - the Virasoro algebra. This is a one-dimensional $\mathbb{k}[T]$ module with generator $v$ such that $v_{(\lambda)} v=(T+2 \lambda) v$.

An important generalization of conformal algebras was proposed in 4 . For every Hopf algebra $H$ with coproduct $\Delta: H \rightarrow H \otimes H$ and antipode $S: H \rightarrow H$ one may consider the class $\mathcal{M}(H)$ of left unital $H$-modules as a pseudo-tensor category in the sense of [3]. The notion of a pseudo-tensor category generalizes the notion of an operad. Namely, operads are exactly the pseudo-tensor category with only one object. For one-dimensional Hopf algebra $H=\mathbb{k}, \mathcal{M}(H)$ is the class of linear spaces equipped by polylinear maps.

An algebra in $\mathcal{M}(H)$ is called a pseudo-algebra over $H$. This is a module $C \in$ $\mathcal{M}(H)$ endowed with a map

$$
*: C \otimes C \rightarrow(H \otimes H) \otimes_{H} C
$$

such that

$$
(h a * g b)=\left((h \otimes g) \otimes_{H} 1\right)(a * b), \quad a, b \in C, h, g \in H .
$$

Here $H \otimes H$ is considered as the outer product of regular right $H$-modules, i.e., $(h \otimes g) \cdot f=(h \otimes g) \Delta(f), f, g, h \in H$.

Conformal algebras in the sense of Definition 1 are exactly pseudo-algebras over $H=\mathbb{k}[T], \Delta(T)=T \otimes 1+1 \otimes T, S(T)=-T$. Namely, if $C$ is a conformal algebra, $a, b \in C,\left(a_{(\lambda)} b\right)=\sum_{n \geq 0} \lambda^{n} c_{n}$ then $a * b=\sum_{n \geq 0}(-1)^{n}\left(T^{n} \otimes 1\right) \otimes_{H} c_{n}$.

From the categorical point of view (similar to [9]), a pseudo-algebra is a functor from the operad of planar binary trees to $\mathcal{M}(H)$. Given a variety $\mathfrak{M}$ of (ordinary) algebras defined by a family of homogeneous polylinear identities, one may define what is an $\mathfrak{M}$-pseudo-algebra over $H$ by means of the operad associated with $\mathfrak{M}$. For conformal algebras, this definition is equivalent to the mentioned above: An $\mathfrak{M}$-pseudo-algebra over $\mathbb{k}[T](\operatorname{char} \mathbb{k}=0)$ is the same as $\mathfrak{M}$-conformal algebra [10].

A systematic study of Lie and associative pseudo-algebras over a wide class of cocommutative Hopf algebras was done in [4].

The operad associated with Lie (or commutative) algebras is symmetric, but $\mathcal{M}(H)$ is a symmetric pseudo-tensor category only if $H$ is cocommutative. This is 
why one cannot define Lie pseudo-algebras over a non-cocommutative Hopf algebras in this way. Nevertheless, associative pseudoalgebras may be defined over an arbitrary Hopf algebra.

A natural class of Hopf algebras is given by coordinate algebras of linear algebraic groups. If $G$ is such a group and $H=\mathbb{k}[G]$ is the algebra of regular functions on $G$ then the class of pseudo-algebras over $H$ can be completely described in terms of operations indexed by elements of $G$.

Definition 2 ([6]). A conformal algebra over $G$ is a left unital $H$-module $C$ endowed with a family of bilinear operations

$$
(\cdot(g) \cdot): C \otimes C \rightarrow C, \quad g \in G
$$

such that the following properties hold.

- For every $a, b \in C$ there exists $\sum_{i} h_{i} \otimes c_{i} \in H \otimes C$ such that $\left(a_{(g)} b\right)=$ $\sum_{i} h_{i}(g) c_{i}$ for all $g \in G$

- $\left(h a_{(g)} b\right)=h\left(g^{-1}\right)\left(a_{(g)} b\right)$;

- $\left(a_{(g)} h b\right)=L_{g} h\left(a_{(g)} b\right)$, where $\left(L_{g} h\right)(x)=h(g x)$ for $x \in G$.

Obviously, if $G=\mathbb{A}^{1} \simeq(\mathbb{k},+)$ then this definition coincides with Definition 1, In general, a conformal algebra over $G$ is the same as pseudo-algebra over $H=\mathbb{k}[G]$. Associativity of such a pseudo-algebra $C$ may be expressed in terms of $g$-products as follows:

$$
a_{(g)}\left(b_{(h)} c\right)=\left(a_{(g)} b\right)_{(h g)} c, \quad a, b, c \in C, g, h \in G .
$$

The main purpose of this paper is to describe the structure of simple and semisimple conformal algebras over linear algebraic groups $G$ such that $G^{0}$ is isomorphic to the affine line. It turns out that such objects are natural to describe in terms of graded conformal algebras over the affine line. This is a step towards complete structure theory of conformal algebras over 1-dimensional linear algebraic groups. Another case (when $G^{0} \simeq \mathbb{k}^{*}$ ) corresponds to $\mathbb{Z}$-conformal algebras introduced in 11. Structure theory of such algebras is a topic for further investigation.

\section{Graded conformal algebras}

Let $G^{0}$ be an abelian connected linear algebraic group, and let Aut $G^{0}$ be the group of its continuous automorphisms. Suppose $\Gamma$ is a group, and fix a homomorphism $\sigma: \Gamma \rightarrow$ Aut $G^{0}$. Also, choose a 2-cocycle $\varphi \in Z^{2}\left(\Gamma, G^{0}, \sigma\right)$, i.e., a map $\varphi: \Gamma \times \Gamma \rightarrow G^{0}$ such that

$$
\varphi(\alpha \beta, \gamma) \varphi(\alpha, \beta)^{\sigma(\gamma)}=\varphi(\alpha, \beta \gamma) \varphi(\beta, \gamma)
$$

for all $\alpha, \beta, \gamma \in \Gamma$ and $\varphi(e, e)=e$. Note that $\varphi(\alpha, e)=\varphi(e, \alpha)=e$ and $\varphi\left(\alpha^{-1}, \alpha\right)=$ $\varphi\left(\alpha, \alpha^{-1}\right)^{\sigma(\alpha)}$ for all $\alpha \in \Gamma$.

Denote by $H^{0}$ the algebra of regular functions on $G^{0}$. For $\lambda \in G^{0}$, denote by $L_{\lambda}$ the shift operator on $H^{0}$, i.e., $\left(L_{\lambda} h\right)(\mu)=h(\lambda \mu), h \in H^{0}, \mu \in G^{0}$.

Definition 3. A $(\Gamma, \sigma, \varphi)$-graded conformal algebra over $G^{0}$ is a graded $H^{0}$-module

$$
C=\bigoplus_{\gamma \in \Gamma} C_{\gamma}
$$

equipped by a family of $\mathbb{k}$-bilinear operations $(\cdot(\lambda) \cdot), \lambda \in G^{0}$, such that: 
(C1) For every $a, b \in C$ the function $\left(a_{(x)} b\right)$ is regular, i.e., $\left(a_{(\lambda)} b\right)=\sum_{i} h_{i}(\lambda) c_{i}$ for an appropriate $\sum_{i} h_{i} \otimes c_{i} \in H^{0} \otimes C$.

(C2) If $a \in C_{\alpha}$ and $b \in C_{\beta}$ then $\left(a_{(\lambda)} b\right) \in C_{\alpha \beta}, \alpha, \beta \in \Gamma, \lambda \in G^{0}$.

(C3) If $a \in C_{\alpha}, b \in C_{\beta}$, and $h \in H^{0}$ then

$$
\begin{gathered}
\left(h a_{(\lambda)} b\right)=h\left(\left(\lambda^{-1} \varphi\left(\alpha, \alpha^{-1}\right)^{-1}\right)^{\sigma(\alpha)}\right)\left(a_{(\lambda)} b\right), \\
\left(a_{(\lambda)} h b\right)=\left(L_{\lambda^{\sigma(\alpha \beta)}} \varphi\left(\alpha^{-1}, \alpha \beta\right)\right. \\
h)\left(a_{(\lambda)} b\right)
\end{gathered}
$$

for all $\lambda \in G^{0}$.

If $C$ is a finitely generated $H^{0}$-module then $C$ is said to be finite $(\Gamma, \sigma, \varphi)$-graded conformal algebra over $G^{0}$.

A homomorphism of $(\Gamma, \sigma, \varphi)$-graded conformal algebras over $G^{0}$ is a homogeneous homomorphism of graded $H^{0}$-modules preserving the operations $(\cdot(\lambda) \cdot)$ for all $\lambda \in G^{0}$.

Recall that 2-cocycles $\varphi, \varphi^{\prime} \in Z^{2}\left(\Gamma, G^{0}, \sigma\right)$ are called cohomological if there exists a 1-cochain $\tau: \Gamma \rightarrow G^{0}$ such that

$$
\varphi^{\prime}(\alpha, \beta) \varphi(\alpha, \beta)^{-1}=\tau(\alpha)^{\sigma(\beta)} \tau(\beta) \tau(\alpha \beta)^{-1}, \quad \alpha, \beta \in \Gamma .
$$

Given $\Gamma$ and $\sigma$ as above, a 2-cocycle $\varphi \in Z^{2}\left(\Gamma, G^{0}, \sigma\right)$ defines an extension

$$
\{e\} \rightarrow G^{0} \rightarrow G \rightarrow \Gamma \rightarrow\{e\},
$$

where $\sigma$ represents the conjugation: $g^{-1} \lambda g=\lambda^{\sigma(\gamma)}$, where $\gamma=g G^{0} \in \Gamma=G / G^{0}$. Cocycles $\varphi$ and $\varphi^{\prime}$ define isomorphic extensions if and only if they are cohomological (see, e.g., [12]).

Proposition 1. If $\varphi, \varphi^{\prime} \in Z^{2}\left(\Gamma, G^{0}, \sigma\right)$ are cohomological then the categories of $(\Gamma, \sigma, \varphi)$ - and $\left(\Gamma, \sigma, \varphi^{\prime}\right)$-graded conformal algebras over $G^{0}$ are equivalent.

Proof. Suppose $C=\bigoplus_{\gamma \in \Gamma} C_{\gamma}$ is a $(\Gamma, \sigma, \varphi)$-graded conformal algebra, and $\varphi^{\prime} \in$ $Z^{2}\left(\Gamma, G^{0}, \varphi\right)$ is related with $\varphi$ by (4). Then we may define a new structure of an $H^{0}$-module and a new family of operations $\left(\cdot[\lambda]^{\cdot}\right)$ on $C$ by the following rule:

$$
h \cdot a=\left(L_{\tau(\alpha)^{-1}} h\right) a, \quad\left(a_{[\lambda]} b\right)=\left(a_{\left(\lambda \tau\left(\alpha^{-1}\right)\right)} b\right)
$$

for $a \in C_{\alpha}, b \in C, \lambda \in G^{0}, h \in H^{0}$. Straightforward computations show this new system is a $\left(\Gamma, \sigma, \varphi^{\prime}\right)$-graded conformal algebra over $G^{0}$ (it is enough to check the condition (C3) of Definition 3). The correspondence constructed is obviously an equivalence of categories.

Example 1. If $G^{0}$ is the trivial group then $\sigma$ and $\varphi$ are also trivial, so Definition 3 describes the class of $\Gamma$-graded ordinary algebras over $\mathbb{k}$.

Example 2. Let $A=\bigoplus_{\gamma \in \Gamma} A_{\gamma}$ be an (ordinary) $\Gamma$-graded algebra. Then the free $H^{0}$ module $C=H^{0} \otimes A$ can be endowed with a natural structure of a $(\Gamma, \sigma, \varphi)$-graded conformal algebra over $G^{0}$ via

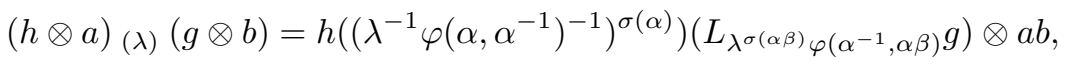

$h, g \in H^{0}, a \in A_{\alpha}, b \in A_{\beta}$. The graded conformal algebra $C$ obtained is called the current conformal algebra over $A$, it is denoted by Cur $A$.

The following example explains the origin of Definition 3 
Example 3. Consider a linear algebraic group $G$ with abelian identity component $G^{0}$. Denote by $\Gamma$ the quotient group $G / G^{0}$. Then there exist $\sigma: \Gamma \rightarrow$ Aut $G^{0}$ and $\varphi \in Z^{2}\left(\Gamma, G^{0}, \sigma\right)$ such that $G$ is isomorphic to the set $\Gamma \times G^{0}$ endowed with the product

$$
(\gamma, \lambda)(\beta, \mu)=\left(\gamma \beta, \lambda^{\sigma(\beta)} \mu \varphi(\gamma, \beta)\right)
$$

$\gamma, \beta \in \Gamma, \lambda, \mu \in G^{0}$.

The Hopf algebra $H=\mathbb{k}[G]$ of regular functions on $G$ may be presented as the algebra $(\mathbb{k} \Gamma)^{*} \otimes H^{0}$ with coproduct and antipode depending on $\sigma$ and $\varphi$. Here $(\mathbb{k} \Gamma)^{*}$ is the dual group algebra of $\Gamma$ spanned by functionals $T_{\gamma}: \Gamma \rightarrow \mathbb{k},\left\langle T_{\gamma}, \beta\right\rangle=\delta_{\gamma, \beta}$, $\gamma, \beta \in \Gamma$.

Suppose $C$ is a conformal algebra over $G$ [6], i.e., a pseudo-algebra over $H$ [4]. The natural decomposition $C=\bigoplus_{\gamma \in \Gamma} C_{\gamma}, C_{\gamma}=\left\{x \in C \mid\left(T_{\gamma} \otimes 1\right) x=x\right\}$, defines a $\Gamma$-grading on the $H^{0}$-module $C$. Moreover, the operations

$$
\left(a_{(\lambda)} b\right)=\left(a_{\left(\alpha^{-1}, \lambda\right)} b\right), \quad a \in C_{\alpha}, b \in C, \lambda \in G^{0},
$$

turn $C$ into a $(\Gamma, \sigma, \varphi)$-graded conformal algebra over $G^{0}$.

Conversely, every $(\Gamma, \sigma, \varphi)$-graded conformal algebra over $G^{0}$ (for a finite group $\Gamma$ ) is a conformal algebra over the corresponding extension of $G^{0}$.

Let $V=\bigoplus_{\gamma \in \Gamma} V_{\gamma}$ be a graded linear space. Then the algebra End $V$ of linear transformations of $V$ carries the following natural grading:

$$
\text { End } V=\bigoplus_{\gamma \in \Gamma}(\text { End } V)_{\gamma} \text {, }
$$

$$
\text { (End } V)_{\gamma}=\left\{\xi \in \text { End } V \mid \xi\left(V_{\alpha}\right) \subseteq V_{\gamma \alpha}, \alpha \in \Gamma\right\} .
$$

A map $a: G^{0} \rightarrow$ End $V$ is called locally regular if for every $v \in V$ there exists an element $\left(a_{(x)} v\right)=\sum_{i} h_{i} \otimes v_{i} \in H^{0} \otimes V$ such that $a(\lambda) v=\sum_{i} f_{i}(\lambda) v_{i}$ for all $\lambda \in G^{0}$.

Example 4. Let $M=\bigoplus_{\gamma \in \Gamma} M_{\gamma}$ be a graded $H^{0}$-module. Denote by $\left(\operatorname{Cend}^{\Gamma} M\right)_{\gamma}$ the space of all locally regular maps $a: G^{0} \rightarrow(\text { End } M)_{\gamma}$ such that

$$
a(\lambda)(h u)=\left(L_{\lambda \sigma(\gamma \beta)} \varphi\left(\gamma^{-1}, \gamma \beta\right) h\right) a(\lambda) u
$$

for all $h \in H^{0}, u \in M_{\beta}, \lambda \in G^{0}$.

In fact, the structure of $\left(\operatorname{Cend}^{\Gamma} M\right)_{\gamma}$ depends also on $\sigma$ and $\varphi$, but we use the single letter $\Gamma$ in order to simplify notations. We will later use Cend without a superscript for the space of "non-graded" conformal linear maps, as in [1].

For each $\gamma \in \Gamma$ the space $\left(\operatorname{Cend}^{\Gamma} M\right)_{\gamma}$ carries the following $H^{0}$-module structure:

$$
(h a): \lambda \rightarrow h\left(\left(\lambda^{-1} \varphi\left(\gamma, \gamma^{-1}\right)^{-1}\right)^{\sigma(\gamma)}\right) .
$$

Consider the formal direct sum of all $\left(\operatorname{Cend}^{\Gamma} M\right)_{\gamma}$ as a graded $H^{0}$-module denoted by $\operatorname{Cend}^{\Gamma} M$. There exists a natural family of bilinear operations $(\cdot(\lambda) \cdot)$ on $\operatorname{Cend}^{\Gamma} M$, namely,

$$
\left(a_{(\lambda)} b\right)(\mu)=a(\lambda) b\left(\left(\lambda^{-1} \mu \varphi\left(\beta^{-1}, \alpha^{-1}\right)^{-1}\right)^{\sigma(\alpha)}\right)
$$

for $a \in\left(\operatorname{Cend}^{\Gamma} M\right)_{\alpha}, b \in\left(\operatorname{Cend}^{\Gamma} M\right)_{\beta}, \lambda, \mu \in G^{0}$. It is easy to check that the operations given by (7) satisfy the conditions (C2) and (C3) of Definition 3 , Moreover, if $M$ is a finitely generated $H^{0}$-module then the condition $(\mathrm{C} 1)$ also holds. 
Indeed, for a fixed system of homogeneous generators $u_{1}, \ldots, u_{n} \in M$, every map $a \in \operatorname{Cend}^{\Gamma} M$ is completely defined by no more than $n^{2}$ regular functions $h_{i j} \in H^{0}$. Indeed, it is enough to consider

$$
a(x) u_{i}=\sum_{j=1}^{n} f_{i j} \otimes u_{j}, \quad i=1, \ldots, n .
$$

Hence, $\left(a_{(x)} b\right)$ can also be considered as an element in $H^{0} \otimes \operatorname{Cend}^{\Gamma} M$.

For every $(\Gamma, \sigma, \varphi)$-graded conformal algebra $C$ over $G^{0}$ there exists a natural homogeneous $H^{0}$-linear map $\ell: C \rightarrow \operatorname{Cend}^{\Gamma} C, \ell(a): \lambda \mapsto\left(a_{(\lambda)} \cdot\right) \in$ End $C, a \in C$, $\lambda \in G^{0}$. If $\ell$ preserves operations $\left(\cdot{ }_{(\lambda)} \cdot\right)$ for all $\lambda \in G^{0}$ then $C$ is said to be associative. This definition is similar to the case of ordinary algebras when Cend means the same as End. Thus, the axiom of associativity for graded conformal algebras has the following form:

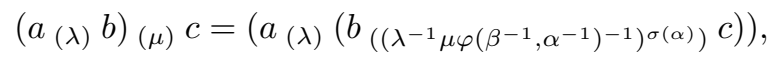

$a \in C_{\alpha}, b \in C_{\beta}, c \in C, \lambda, \mu \in G^{0}$.

In particular, if $A$ is an associative graded algebra then so is Cur $A$, if $M$ is a finitely generated graded $H^{0}$-module then $\operatorname{Cend}^{\Gamma} M$ is an associative graded conformal algebra.

Let $M=\bigoplus_{\gamma \in \Gamma} M_{\gamma}$ be a finitely generated graded $H^{0}$-module. A representation $\rho$ of an associative $(\Gamma, \sigma, \varphi)$-graded conformal algebra $C$ over $G^{0}$ on $M$ is a homomorphism $\rho: C \rightarrow \operatorname{Cend}^{\Gamma} M$ of $(\Gamma, \sigma, \varphi)$-graded conformal algebras over $G^{0}$. Given a representation of $C$ on $M$, the latter is called a graded conformal $C$-module.

As in the case of ordinary algebras, we use the following convention: $\rho(a)(\lambda) v=$ $a_{(\lambda)} v, a \in C, v \in M$. If $\rho$ is injective then the $C$-module $M$ is said to be faithful; if $\rho \neq 0$ and there are no nonzero proper graded $C$-invariant $H^{0}$-submodules in $M$ then $M$ is called irreducible. Irreducibility of $M$ means that for every $0 \neq v \in M_{\gamma}$ and for every $u \in M_{\alpha \gamma}(\alpha, \gamma \in \Gamma)$ there exist $h_{i} \in H^{0}, a_{i} \in C_{\alpha}, \lambda_{i} \in G^{0}, i=$ $1, \ldots, n$, such that

$$
u=\sum_{i=1}^{n} h_{i}\left(a_{i}\left(\lambda_{i}\right) v\right)
$$

Definition 4. A graded subalgebra $C$ of the $(\Gamma, \sigma, \varphi)$-graded conformal algebra Cend $^{\Gamma} M$ is irreducible if $M$ is an irreducible graded conformal $C$-module.

If $C_{(\lambda)} C \neq 0$ for some $\lambda \in G^{0}$ and $C$ has no non-zero proper graded ideals then $C$ is said to be simple. A graded ideal $I$ of $C$ is abelian if $I{ }_{(\lambda)} I=0$ for all $\lambda \in G^{0}$. If $C$ has no nonzero abelian graded ideals then $C$ is called semisimple.

Lemma 1. Let $C$ be a $(\Gamma, \sigma, \varphi)$-graded associative conformal algebra over $G^{0}, U$ be a graded conformal $C$-module, and let $W \subseteq U$ be a graded $C$-submodule such that $h U \subseteq W$ for some $h \in H^{0}$. Then $\left(C_{(\lambda)} U\right) \subseteq W$ for all $\lambda \in G^{0}$.

Proof. Consider an arbitrary pair of homogeneous elements $a \in C_{\alpha}, u \in U_{\beta}$, where $\alpha, \beta \in \Gamma$. Suppose $\left(a_{(x)} u\right)=\sum_{i} h_{i} \otimes v_{i} \in H^{0} \otimes U$. Then

$$
\left(a_{(x)} h u\right)=\sum_{i,(h)} h_{i} s_{\alpha, \beta}\left(h_{(1)}\right) \otimes h_{(2)} v_{i}
$$


where $\sum_{(h)} h_{(1)} \otimes h_{(2)}$ is the Sweedler's notation for the standard coproduct in $H^{0}$, $s_{\alpha, \beta}$ is the following automorphism of the algebra $H^{0}$ :

$$
s_{\alpha, \beta}(f): \lambda \mapsto\left(L_{\varphi\left(\alpha^{-1}, \alpha \beta\right)} f\right)\left(\lambda^{\sigma(\alpha \beta)}\right), \quad f \in H^{0}, \lambda \in G^{0} .
$$

Consider the linear map $\Psi_{\alpha, \beta}: H^{0} \otimes U \rightarrow H^{0} \otimes U$ defined as follows:

$$
\Psi_{\alpha, \beta}(f \otimes v)=\sum_{(f)} s_{\alpha, \beta}\left(f_{(1)}\right) \otimes f_{(2)} v, \quad v \in U .
$$

This map is invertible since

$$
\Psi_{\alpha, \beta}^{-1}(f \otimes v)=\sum_{\left(s_{\alpha, \beta}^{-1}(f)\right)}\left(s_{\alpha, \beta}^{-1}(f)\right)_{(1)} \otimes S\left(\left(s_{\alpha, \beta}^{-1}(f)\right)_{(2)}\right) v
$$

where $S$ is the standard antipode on $H^{0}$.

Since $\left(a_{(x)} h u\right) \in H^{0} \otimes W \subset H^{0} \otimes U$, we also have

$$
\Psi_{\alpha, \beta}^{-1}\left(a_{(x)} h u\right)=(h \otimes 1) \Psi_{\alpha, \beta}^{-1}\left(a_{(x)} u\right) \in H^{0} \otimes W .
$$

The group $G^{0}$ is connected and $H^{0}$ has no zero divisors. Therefore, (9) implies $\Psi_{\alpha, \beta}^{-1}\left(a_{(x)} u\right) \in H^{0} \otimes W$. By the definition of $\Psi_{\alpha, \beta},\left(a_{(x)} u\right) \in H^{0} \otimes W$.

Proposition 2. Let $C$ be a finite simple $(\Gamma, \sigma, \varphi)$-graded conformal algebra over $G^{0}$. Then there exists a finite faithful irreducible graded conformal $C$-module $M$ which is a torsion-free $H^{0}$-module.

Proof. Since $H^{0}$ is a Noetherian algebra, $C$ is a Noetherian $H^{0}$-module. There exists a maximal graded left ideal $I$ of $C$. Then $M=C / I$ is an irreducible graded conformal $C$-module which is obviously faithful.

Moreover, $M$ is a torsion-free $H^{0}$-module. Indeed, consider the graded left ideal $I_{1}=\left\{a \in C \mid a+I \in \operatorname{Tor}_{H^{0}}(C / I)\right\}$ which contains $I$. Since $I$ is maximal, we have either $I_{1}=I$ or $I_{1}=C$. The first option means $\operatorname{Tor}_{H^{0}}(C / I)=0$, so assume the second holds.

In this case, there exists $h \in H^{0} \backslash\{0\}$ such that $h C \subseteq I$. By Lemma 1 $\left(C_{(\lambda)} C\right) \subseteq$ $I$ for all $\lambda \in G^{0}$, which is impossible for a simple algebra.

Proposition 3. Let $C$ be an irreducible graded subalgebra of the $(\Gamma, \sigma, \varphi)$-graded conformal algebra $\operatorname{Cend}^{\Gamma} M$ (over $G^{0}$ ) for a finitely generated graded $H^{0}$-module $M$. Then the homogeneous component $C_{e}$ is a semisimple conformal algebra over $G^{0}$.

Proof. For an arbitrary $0 \neq v \in M_{\gamma}$, the irreducibility of $C$ implies

$$
C_{e} v:=H^{0}\left\{a_{(\lambda)} v \mid a \in C_{e}, \lambda \in G^{0}\right\}=M_{\gamma} .
$$

Suppose $I$ is an abelian ideal of $C_{e}, I \neq 0$. If $0 \neq I v \neq M_{\gamma}$ then $C_{e}(I v) \subseteq I v \neq M_{\gamma}$ which contradicts the irreducibility of $M$. Therefore, for each $\gamma \in \Gamma$ either $I M_{\gamma}=0$ or $I M_{\gamma}=M_{\gamma}$. Since $M$ is faithful and $I \neq 0$, there exists at least one $\gamma \in \Gamma$ such that $M_{\gamma} \neq 0$ and $I M_{\gamma}=M_{\gamma}$. But then $M_{\gamma}=I\left(I M_{\gamma}\right)=0$, a contradiction. 


\section{IRREDUCIBLE GRADED ALGEBRAS OF LINEAR TRANSFORMATIONS}

Suppose $V=\bigoplus_{\gamma \in \Gamma} V_{\gamma}$ is a finite-dimensional $\Gamma$-graded linear space. Then End $V$ is a $\Gamma$-graded associative algebra with respect to the natural grading (5). In this section, we consider the following problem: Describe graded irreducible subalgebras of End $V$, i.e., graded subalgebras $A \subseteq$ End $V$ such that $V$ has no nonzero proper graded $A$-invariant subspaces. (In the non-graded settings, the answer is given by the classical Burnside Theorem.) The case $\Gamma=\mathbb{Z}_{2}$ was considered in 13. If $\Gamma$ is a finite group then this problem naturally fits within the frames of conformal algebras over $\Gamma$; it was solved in $[6$. Here we consider the general case.

Without loss of generality, we may assume $V_{e} \neq 0$. Otherwise, we may shift the grading on $V$ by setting $V_{\gamma}^{\prime}=V_{\gamma g}$ for a fixed element $g \in \Gamma$. The corresponding grading on End $V$ and its graded irreducible subalgebras remain the same.

In the case $|\Gamma|<\infty$, this problem was solved in [6] in the context of conformal algebras over $\Gamma$. If $\Gamma$ is an infinite group then $(\mathbb{k} \Gamma)^{*}$ is not a Hopf algebra, but we may still obtain a very similar result.

Let $T_{\gamma}, \gamma \in \Gamma$, stands for the projection $V \rightarrow V_{\gamma}$ relative to the given grading on $V$. Denote by $\Gamma_{0}$ the set of all $\gamma \in \Gamma$ such that $V_{\gamma}=0$. The identity operator $\sum_{\gamma \in \Gamma} T_{\gamma}$ is actually presented by finite sum since almost all $T_{\gamma}$ are zero.

A subgroup $\Gamma_{1} \leq \Gamma$ is said to be fine if

$$
\Gamma \backslash \Gamma_{0}=\Gamma_{1} \cup \Gamma_{2} \cup \cdots \cup \Gamma_{p}, \quad \Gamma_{k}=\gamma_{k} \Gamma_{1} .
$$

Let us fix the system of representatives $\gamma_{1}, \ldots, \gamma_{p} \in \Gamma$ assuming $\gamma_{1}=e$.

Denote by $Z\left(\Gamma, \Gamma_{0}, \Gamma_{1}\right)$ the set of all maps $\chi: \Gamma \times \Gamma \rightarrow \mathbb{k}$ such that:

$$
\begin{gathered}
\chi(\gamma, \beta)=0, \quad \beta \in \Gamma_{0} \text { or } \gamma \beta \in \Gamma_{0}, \\
\chi\left(\gamma, \alpha \gamma_{k}\right) \chi(\gamma \alpha, \beta)=\chi(\gamma, \alpha \beta) \chi(\alpha, \beta), \quad \alpha, \gamma \in \Gamma, \beta \in \Gamma_{k},
\end{gathered}
$$

and for $\gamma, \beta \notin \Gamma_{0}$ we have

$$
\begin{gathered}
\chi(\gamma, \beta)=1, \quad \beta=\gamma_{k} \text { or } \gamma=e, \\
\chi(\gamma, \beta) \neq 0 \quad \text { otherwize. }
\end{gathered}
$$

For example, if $\Gamma_{1}=\Gamma$ then $Z\left(\Gamma, \Gamma_{0}, \Gamma_{1}\right)=Z^{2}\left(\Gamma, \mathbb{k}^{*}\right)$. A restriction of $\chi \in$ $Z\left(\Gamma, \Gamma_{0}, \Gamma_{1}\right)$ to $\Gamma_{1} \times \Gamma_{1}$ is a 2 -cocycle from $Z^{2}\left(\Gamma_{1}, \mathbb{k}^{*}\right)$. Moreover, for every $\theta \in$ $Z^{2}\left(\Gamma_{1}, \mathbb{k}^{*}\right)$ such that $\theta(e, e)=1$ the function

$$
\chi(\gamma, \beta)= \begin{cases}\theta\left(\gamma_{q}^{-1} \gamma \gamma_{k}, \gamma_{k}^{-1} \beta\right), & \beta \in \Gamma_{k}, \gamma \beta \in \Gamma_{q}, \\ 0, & \text { otherwize, }\end{cases}
$$

belongs to $Z\left(\Gamma, \Gamma_{0}, \Gamma_{1}\right)$.

Theorem 1. Let $A$ be a graded irreducible subalgebra of End $V$. Then there exist:

- A fine subgroup $\Gamma_{1} \leq \Gamma$ with a family of $\gamma_{k} \in \Gamma, k=1, \ldots, p$, satisfying (10);

- A function $\chi \in Z\left(\Gamma, \Gamma_{0}, \Gamma_{1}\right)$;

- A family of linear isomorphisms $\iota_{\gamma}: V_{\gamma_{k}} \rightarrow V_{\gamma}, \gamma \in \Gamma_{k}$, where $\iota_{\gamma_{k}}=\mathrm{id}_{V_{\gamma_{k}}}$, such that

$$
A_{\alpha}=\left\{\sum_{k=1}^{p} \sum_{\gamma \in \Gamma_{k}} \chi(\alpha, \gamma) \iota_{\alpha \gamma} \iota_{\alpha \gamma_{k}}^{-1} a_{k} \iota_{\gamma}^{-1} T_{\gamma} \mid a_{k} \in \operatorname{Hom}\left(V_{\gamma_{k}}, V_{\alpha \gamma_{k}}\right)\right\}, \quad \alpha \in \Gamma .
$$


The isomorphisms $\iota_{\alpha \gamma}$ and $\iota_{\alpha \gamma_{k}}$ in the right-hand side of (12) are defined only when $\alpha \Gamma_{k} \nsubseteq \Gamma_{0}$. Otherwise, $\chi(\alpha, \gamma)=0$, so we just do not need to take into consideration those summands corresponding to such index $k$.

Proof. Proposition 3 applied to $G^{0}=\{e\}$ implies the algebra $A_{e}$ is semisimple. By the Wedderburn Theorem, $A_{e}=I_{1} \oplus \cdots \oplus I_{p}$, where $I_{k} \simeq M_{n_{k}}(\mathbb{k})$. For every $a \in A_{e}$ we have

$$
a\left(\sum_{\beta \in \Gamma} T_{\beta}\right)=\sum_{\gamma \in \Gamma \backslash \Gamma_{0}} \pi_{\gamma}(a),
$$

where the map $\pi_{\gamma}: A_{e} \rightarrow$ End $V_{\gamma}$ is defined by $\pi_{\gamma}(a)=a T_{\gamma}$. This is a homomorphism of algebras.

For each $k=1, \ldots, p$ set $\Gamma_{k}=\left\{\gamma \in \Gamma \mid \pi_{\gamma}\left(I_{k}\right) \neq 0\right\} \neq \emptyset$. Obviously, $\Gamma \backslash \Gamma_{0}=$ $\Gamma_{1} \cup \cdots \cup \Gamma_{p}, \Gamma_{i} \cap \Gamma_{j}=\emptyset$ for $i \neq j$.

Let us fix a system of representatives $\gamma_{k} \in \Gamma_{k}$ for $k=1, \ldots, p$, and assume $e \in \Gamma_{1}, \gamma_{1}=e$. If $\gamma \in \Gamma_{k}$ then $\left(\operatorname{dim} V_{\gamma}\right)^{2}=\operatorname{dim} I_{k}$, so $n_{\gamma}=n_{k}$. Moreover, $\pi_{\gamma}\left(I_{k}\right)=$ End $V_{\gamma}$. Hence, $\pi_{\gamma} \pi_{\gamma_{k}}^{-1}$ : End $V_{\gamma_{k}} \rightarrow$ End $V_{\gamma}$. is also an isomorphism, so it can be presented by conjugation: There exist linear isomorphisms $\iota_{\gamma}: V_{\gamma_{k}} \rightarrow V_{\gamma}$, $\gamma \in \Gamma_{k}, k=1, \ldots, p$, such that $\pi_{\gamma} \pi_{\gamma_{k}}^{-1}(a)=\iota_{\gamma} a \iota_{\gamma}^{-1}$ for $a \in$ End $V_{\gamma_{k}}$.

Finally, (13) implies

$$
A_{e}=\left\{\sum_{k=1}^{p} \sum_{\gamma \in \Gamma_{k}} \iota_{\gamma} a_{k} \iota_{\gamma}^{-1} T_{\gamma} \mid a_{k} \in \operatorname{End} V_{\gamma_{k}}\right\} .
$$

Lemma 2. The subsets $\Gamma_{k} \subseteq \Gamma, k=1, \ldots, p$, are left adjacent classes in $\Gamma$ with respect to finite subgroup $\Gamma_{1}$.

Proof. It is enough to show the following property: $\gamma \Gamma_{k} \cap \Gamma_{m} \neq \emptyset$ implies $\Gamma_{m} \subseteq \gamma \Gamma_{k}$ for every $\gamma \in \Gamma$ and for every $k, m \in\{1, \ldots, p\}$. Indeed, the same property for $\gamma^{-1}$ would show $\gamma \Gamma_{k}$ is equal to $\Gamma_{m}$ provided they have nonempty intersection. Hence, the finite set $\Gamma_{1}$ is a subgroup, and all $\Gamma_{k}, k=1, \ldots, p$, are left adjacent classes in $\Gamma$.

Suppose $\gamma_{0} \in \gamma \Gamma_{k} \cap \Gamma_{m}, \gamma_{0}=\gamma \beta_{0}, \beta_{0} \in \Gamma_{k}$. We may choose $0 \neq u \in V_{\beta_{0}}$, $0 \neq v \in V_{\gamma_{0}}$. Since $A$ is graded irreducible, there exists $a \in A_{\gamma}, b \in A_{\gamma^{-1}}$ such that $a u=v, b v=u$.

If we choose $a_{j}=0$ for $j \neq k$ and $a_{k}=\operatorname{id}_{V_{\gamma_{k}}}$ in (14) then

$$
e_{k}=\sum_{\gamma \in \Gamma_{k}} T_{\gamma} \in A_{e}
$$

Consider $c=a e_{k} b \in A_{e}$. Since $c v=a e_{k}(b v)=a e_{k} u=v, c \neq 0$. Assume $\Gamma_{m} \nsubseteq \gamma \Gamma_{k}$ and choose any $\beta \in \Gamma_{m} \backslash \gamma \Gamma_{k}$. Then for every $w \in V_{\beta}$ we have $c w=a e_{k}(b w)=0$ because $b w \in V_{\gamma^{-1} \beta}, \gamma^{-1} \beta \notin \Gamma_{k}, e_{k}(b w)=0$. The element $c \in A_{e}$ constructed annihilates all $V_{\beta}$ for $\beta \in \Gamma_{m} \backslash \gamma \Gamma_{k}$, but $c v=v \neq 0$ for $v \in V_{\gamma_{0}}, \gamma_{0} \in \Gamma_{m}$. This is a contradiction to (14).

Consider an arbitrary $a \in A_{\alpha}, \alpha \in \Gamma$. Then

$$
a=\sum_{\gamma \in \Gamma} a_{\gamma}, \quad a_{\gamma}=a T_{\gamma} \in \operatorname{Hom}\left(V_{\gamma}, V_{\alpha \gamma}\right) .
$$


Here $a_{\gamma} \in \operatorname{Hom}\left(V_{\gamma}, V_{\alpha \gamma}\right)$. Denote $a_{\gamma_{k}}$ by $a_{k}, k=1, \ldots, p$. Note that if $\gamma \in \Gamma_{k}$, $\alpha \gamma \in \Gamma_{m}$ then $e_{m} a e_{k} \in A_{\alpha}$, so $\sum_{\gamma \in \Gamma_{k}} a_{\gamma} \in A_{\alpha}$ for every $k=1, \ldots, p$.

If $\alpha \gamma_{k} \notin \Gamma_{0}$ then $W_{\alpha, \gamma}=\left\{b \in \operatorname{Hom}\left(V_{\gamma}, V_{\alpha \gamma}\right) \mid \exists a \in A_{\alpha}: a_{\gamma}=b\right\} \neq 0$ for every $\gamma \in \Gamma_{k}$. Since $W_{\alpha, \gamma}$ is a (End $\left.V_{\alpha \gamma}\right)$-(End $\left.V_{\gamma}\right)$-bimodule embedded into $\operatorname{Hom}\left(V_{\gamma}, V_{\alpha \gamma}\right)$, we have $W_{\alpha, \gamma}=\operatorname{Hom}\left(V_{\gamma}, V_{\alpha \gamma}\right)$.

Given $\gamma \in \Gamma_{k}, \alpha \in \Gamma, \alpha \Gamma_{k}=\Gamma_{m}$, the map $\sigma_{\alpha, \gamma}: a_{k} \mapsto a_{\gamma}, a \in A_{\alpha}$, is a linear bijection between $\operatorname{Hom}\left(V_{\gamma_{k}}, V_{\alpha \gamma_{k}}\right)$ and $\operatorname{Hom}\left(V_{\gamma}, V_{\alpha \gamma}\right)$. Assume $\sigma_{\alpha, \gamma_{k}}=$ id and $\sigma_{e, \gamma}(x)=\iota_{\gamma} x \iota_{\gamma}^{-1}$, since we have already established the structure of $A_{e}$.

Suppose $a=\sum_{\gamma \in \Gamma_{k}} a_{k}^{\sigma_{\alpha, \gamma}} \in A_{\alpha}, b=\sum_{\beta \in \Gamma_{m}} \iota_{\beta} b_{m} \iota_{\beta}^{-1} T_{\beta} \in A_{e}$, and consider

$$
b a=\sum_{\gamma \in \Gamma_{k}} \iota_{\alpha \gamma} b_{m} \iota_{\alpha \gamma} a_{k}^{\sigma_{\alpha, \gamma}} .
$$

Here $(b a)_{k}=\iota_{\alpha \gamma_{k}} b_{m} \iota_{\alpha \gamma_{k}}^{-1} a_{k},(b a)_{\gamma}=\iota_{\alpha \gamma} b_{m} \iota_{\alpha \gamma}^{-1} a_{k}^{\sigma_{\alpha, \gamma}}$. Similarly, if

$$
c=\sum_{\beta \in \Gamma_{k}} \iota_{\beta} c_{k} \iota_{\beta}^{-1} T_{\beta} \in A_{e}
$$

then $(a c)_{k}=a_{k} c_{k},(a c)_{\gamma}=a_{k}^{\sigma_{\alpha, \gamma}} \iota_{\gamma} c_{k} \iota_{\gamma}^{-1}$.

Summarizing, if $a \in \operatorname{Hom}\left(V_{\gamma_{k}}, V_{\alpha \gamma_{k}}\right)$ then

$$
(b a c)^{\sigma_{\alpha, \gamma}}=\iota_{\alpha \gamma} \iota_{\alpha \gamma_{k}}^{-1} b \iota_{\alpha \gamma_{k}} \iota_{\alpha \gamma}^{-1} a^{\sigma_{\alpha, \gamma}} \iota_{\gamma} c \iota_{\gamma}^{-1}
$$

for all $b \in$ End $V_{\alpha \gamma_{k}}, c \in$ End $V_{\gamma_{k}}$. Define the linear transformation $\sigma_{\alpha, \gamma}^{\prime}$ of $\operatorname{Hom}\left(V_{\gamma_{k}}, V_{\alpha \gamma_{k}}\right)$ by the following rule:

$$
\sigma_{\alpha, \gamma}^{\prime}: x \mapsto \iota_{\alpha \gamma_{k}} \iota_{\alpha \gamma}^{-1} x^{\sigma_{\alpha, \gamma}} \iota_{\gamma}, \quad x \in \operatorname{Hom}\left(V_{\gamma_{k}}, V_{\alpha \gamma_{k}}\right) .
$$

Then $(b a c)^{\sigma_{\alpha, \gamma}^{\prime}}=b a^{\sigma_{\alpha, \gamma}^{\prime}} c$ for all $b \in$ End $V_{\alpha \gamma_{k}}, c \in$ End $V_{\gamma_{k}}$. Hence, $x^{\sigma_{\alpha, \gamma}^{\prime}}=$ $\chi(\alpha, \gamma) x$, where $\chi: \Gamma \times \Gamma \rightarrow \mathbb{k}$, and we may assume $\chi(\alpha, \gamma)=0$ for $\gamma \in \Gamma_{0}$ or $\alpha \gamma \in$ $\Gamma_{0}$. Also, $\chi\left(\alpha, \gamma_{k}\right)=1, \chi(e, \gamma)=1$ for $\gamma \notin \Gamma_{0}$. Finally, $x^{\sigma_{\alpha, \gamma}}=\chi(\alpha, \gamma) \iota_{\alpha \gamma} \iota_{\alpha \gamma_{k}}^{-1} x \iota_{\gamma}^{-1}$, which implies (12).

It remains to derive (11). Consider two elements $a \in A_{\alpha}, b \in A_{\beta}$, where

$$
a=\sum_{\gamma \in \Gamma_{k}} \chi(\alpha, \gamma) \iota_{\alpha \gamma} \iota_{\alpha \gamma_{k}}^{-1} a_{k} \iota_{\gamma}^{-1} T_{\gamma}, \quad b=\sum_{\delta \in \Gamma_{m}} \chi(\beta, \delta) \iota_{\beta \delta} \iota_{\beta \gamma_{m}}^{-1} b_{m} \iota_{\delta}^{-1} T_{\delta} .
$$

If $c=a b \neq 0$ then $\beta \Gamma_{m}=\Gamma_{k}, \alpha \Gamma_{k} \nsubseteq \Gamma_{0}$. Straightforward computation shows

$$
c=\sum_{\delta \in \Gamma_{m}} \chi(\alpha, \beta \delta) \chi(\beta, \delta) \iota_{\alpha \beta \delta} \iota_{\alpha \beta \gamma_{m}}^{-1}\left(\iota_{\alpha \beta \gamma_{m}} \iota_{\alpha \gamma_{k}}^{-1} a_{k} \iota_{\beta \gamma_{m}}^{-1} b_{m}\right) \iota_{\delta}^{-1} T_{\delta} .
$$

On the other hand,

$$
c=\sum_{\delta \in \Gamma_{m}} \chi(\alpha \beta, \delta) \iota_{\alpha \beta \delta} \iota_{\alpha \beta \gamma_{m}}^{-1} c_{m} \iota_{\delta}^{-1} T_{\delta},
$$

so for $\delta=\gamma_{m}$ we have

$$
c_{m}=\chi\left(\alpha, \beta \gamma_{m}\right)\left(\iota_{\alpha \beta \gamma_{m}} \iota_{\alpha \gamma_{k}}^{-1} a_{k} \iota_{\beta \gamma_{m}}^{-1} b_{m}\right) .
$$

Therefore, $\chi(\alpha \beta, \delta) \chi\left(\alpha, \beta \gamma_{m}\right)=\chi(\alpha, \beta \delta) \chi(\beta, \delta)$.

Corollary 1. A graded irreducible subalgebra of End $V$ is simple. 
Proof. Suppose $A$ is a graded irreducible subalgebra of End $V$ as described by Theorem 1 $n=n_{1}+\cdots+n_{p}$. The basis of this algebra over $\mathbb{k}$ consists of

$$
X_{(m, i),(k, j)}(\gamma)=\sum_{\beta \in \Gamma_{k}} \chi(g, \beta) \iota_{g \beta} \iota_{g \gamma_{k}}^{-1} E_{i j} \iota_{\beta}^{-1} T_{\beta}, \quad g=\gamma_{m} \gamma \gamma_{k}^{-1},
$$

where $k, m=1, \ldots, p, i=1, \ldots, n_{m}, j=1, \ldots, n_{k}, \gamma \in \Gamma_{1}$, and $E_{i j}: V_{\gamma_{k}} \rightarrow V_{\gamma_{m}}$ are linear transformations corresponding to matrix units in some fixed homogeneous basis of $V$.

Consider the space of matrices $M_{n}\left(\mathbb{k} \Gamma_{1}\right)$ over the group algebra $\mathbb{k} \Gamma_{1}$. Let us enumerate rows and columns of these matrices by pairs $(k, j), k=1, \ldots, p, j=$ $1, \ldots, n_{k}$, instead of natural numbers $1, \ldots, n$. Given a 2 -cocycle $\chi \in Z^{2}\left(\Gamma_{1}, \mathbb{k}^{*}\right)$, denote by $\mathbb{k}^{\chi} \Gamma_{1}$ the same space $\mathbb{k} \Gamma_{1}$ endowed with twisted multiplication $\gamma \cdot \beta=$ $\chi(\gamma, \beta) \gamma \beta, \gamma, \beta \in \Gamma_{1}$. This graded algebra as well as matrix algebras $M_{n}\left(\mathbb{k}^{\chi} \Gamma_{1}\right)$ with natural grading are known to be simple, see, e.g., 14.

Let $\Phi$ stands for the linear bijection from $M_{n}\left(\mathbb{k} \Gamma_{1}\right)$ to $A$ defined by

$$
\Phi: E_{(m, i),(k, j)} \gamma \mapsto \chi\left(\gamma_{m}, \gamma\right) X_{(m, i),(k, j)}(\gamma), \quad \gamma \in \Gamma_{1} .
$$

Straightforward computation shows that $\Phi$ is an isomorphism of graded algebras $M_{n}\left(\mathbb{k}^{\chi} \Gamma_{1}\right)$ and $A$.

In particular, we obtain one more proof of the structure theorem for finitedimensional graded algebras over an algebraically closed field: Proposition 2 and Corollary 1 immediately imply

Corollary 2 ([14, 15]). A finite-dimensional simple graded associative algebra $A$ over an algebraically closed field is isomorphic to $M_{n}\left(\mathbb{k}^{\chi} \Gamma_{1}\right)$, where $\chi \in Z^{2}\left(\Gamma_{1}, \mathbb{k}^{*}\right)$.

\section{Graded Conformal algebras over the affine line}

In this section, we consider graded conformal algebras over $G^{0}=\mathbb{A}^{1}$, where $\mathbb{A}^{1}$ is the additive group of the base field $\mathbb{k}$ which is assumed to be algebraically closed and char $\mathbb{k}=0$. In this case, $H^{0}=\mathbb{k}[T]$, Aut $G^{0}=\mathbb{k}^{*}$, and a homomorphism $\sigma: \Gamma \rightarrow$ Aut $G^{0}$ maps $\gamma \in \Gamma$ to a nonzero scalar $\sigma(\gamma) \in \mathbb{k}^{*}$, it acts on $\mathbb{A}^{1}$ by multiplication.

Remark 1. If $\Gamma$ is a finite group then for every $\sigma: \Gamma \rightarrow \mathbb{k}^{*}$ all cocycles in $Z^{2}(\Gamma, \mathbb{k}, \sigma)$ are cohomological to the zero cocycle (see [12, III.10.2]). Therefore, to classify finite simple associative conformal algebras over a linear algebraic group $G$ such that $G^{0} \simeq \mathbb{A}^{1}$ it is enough to consider only $(\Gamma, \sigma, 0)$-graded conformal algebras.

However, if $\Gamma$ is infinite then there may exist nontrivial cocycles. This case does not correspond to a linear algebraic group, but we still can consider graded conformal algebras in these settings.

By Proposition 2, to classify simple finite $(\Gamma, \sigma, \varphi)$-graded associative conformal algebras it is enough to describe simple finite graded irreducible subalgebras of the $(\Gamma, \sigma, \varphi)$-graded conformal algebra $\operatorname{Cend}^{\Gamma} M$, where $M=\bigoplus_{\gamma \in \Gamma} M_{\gamma}$ is a finitely generated torsion-free $H^{0}$-module. Such a module over $\mathbb{k}[T]$ is free.

Let us state in details the structure of $\operatorname{Cend}^{\Gamma} M$ in the graded case. Suppose $N$ is the rank of $M$ over $H^{0}$, and let $e_{1}, \ldots, e_{N}$ be a homogeneous basis of $M$. For each $i=1, \ldots, N$ there exists $\alpha_{i} \in \Gamma$ such that $e_{i} \in M_{\alpha_{i}}$. An arbitrary element $a \in \operatorname{Cend}^{\Gamma} M$ is completely defined by the family of regular functions $\left(a_{(\lambda)} e_{i}\right)=$ 
$\sum_{s \geq 0} \sum_{j=1}^{N}(-\lambda)^{s} a_{i j}^{s}(T) e_{j}, i=1, \ldots, N$, where $a_{i j}^{s}(T) \in H^{0}$. Hence, $a \in \operatorname{Cend}^{\Gamma} M$ can be identified with the matrix $[a]=\left(a_{i j}(T, x)\right) \in M_{N}(\mathbb{k}[T, x])$, where

$$
a_{i j}(T, x)=\sum_{s \geq 0}\left(\sigma\left(\alpha_{j} \alpha_{i}^{-1}\right) T+\varphi\left(\alpha_{i} \alpha_{j}^{-1}, \alpha_{j} \alpha_{i}^{-1}\right)\right)^{s} a_{i j}^{s}(x) .
$$

The $H^{0}$-module Cend $^{\Gamma} M$ carries the natural $\Gamma$-grading given by

$$
\left(\operatorname{Cend}^{\Gamma} M\right)_{\gamma}=\sum_{i, j: \gamma=\alpha_{i} \alpha_{j}^{-1}} \mathbb{k}(T, x) E_{i j},
$$

where $E_{i j}$ are the matrix units.

The operations $(\cdot(\lambda) \cdot), \lambda \in \mathbb{A}^{1}$, on matrices over $\mathbb{k}[T, x]$ may be explicitly computed by (7): If $a=f(T, x) E_{i j}, b=g(T, x) E_{j k}$ then

$$
\begin{aligned}
\left(a_{(\lambda)} b\right)=f( & \left.-\sigma(\alpha)\left(\lambda+\varphi\left(\alpha, \alpha^{-1}\right)\right), x\right) \\
& \times g\left(T+\sigma(\alpha \beta) \lambda+\varphi\left(\alpha^{-1}, \alpha \beta\right), x+\sigma\left(\alpha_{i}\right) \lambda+\varphi\left(\alpha^{-1}, \alpha_{i}\right)\right) E_{i k},
\end{aligned}
$$

where $\alpha=\alpha_{i} \alpha_{j}^{-1}, \beta=\alpha_{j} \alpha_{k}^{-1}$.

Let $V=\bigoplus_{\gamma \in \Gamma} V_{\gamma}$ be an $N$-dimensional $\Gamma$-graded linear space, $\operatorname{dim} V_{\gamma}=n_{\gamma}$, and let $M=H^{0} \otimes V$ be the free module with the inherited grading, $M_{\gamma}=H^{0} \otimes V_{\gamma}$.

Given a homogeneous basis $e=\left(e_{1}, \ldots, e_{N}\right)$ of $V$, consider $\operatorname{Cend}_{N}^{\Gamma}=\left\{[a]_{e} \mid\right.$ $\left.a \in \operatorname{Cend}^{\Gamma} M\right\} \simeq M_{N}(\mathbb{k}[T, x])$ as a $(\Gamma, \sigma, \varphi)$-graded conformal algebra in the matrix form (the grading is completely defined by $\alpha_{1}, \ldots, \alpha_{N} \in \Gamma$, as above).

Suppose there is another homogeneous $H^{0}$-basis $f=\left(f_{1}, \ldots, f_{N}\right)$ in $M$ such that $\left(f_{1}, \ldots, f_{N}\right)=\left(e_{1}, \ldots, e_{N}\right) Q(T), Q$ is an invertible matrix in $M_{N}(\mathbb{k}[T])$ which can be presented in a block-diagonal form $Q(T)=\bigoplus_{\gamma \in \Gamma \backslash \Gamma_{0}} Q_{\gamma}(T), Q_{\gamma}, Q_{\gamma}^{-1} \in M_{n_{\gamma}}(\mathbb{k}[T])$.

This is straightforward to compute that for a given $a \in\left(\operatorname{Cend}^{\Gamma} M\right)$ its matrix in the new basis can be found as follows: $[a]_{f}=Q^{-1}(x)[a]_{e} Q^{\Gamma}(T, x)$, where $Q^{\Gamma}(T, x)=$ $\bigoplus_{\gamma \in \Gamma \backslash \Gamma_{0}} Q_{\gamma}(x-\sigma(\gamma) T)$.

Theorem 2. A finite graded irreducible conformal subalgebra $C \subseteq \operatorname{Cend}_{N}^{\Gamma}$ is isomorphic to the current algebra Cur $A$ over a graded irreducible subalgebra $A \subseteq$ End $V$.

Proof. Denote by $\Gamma_{0}$ the set of all $\gamma \in \Gamma$ such that $V_{\gamma}=0$. For each $\gamma \in \Gamma \backslash \Gamma_{0}$ one may consider $M_{\gamma}$ as an ordinary $H^{0}$-module and define the (non-graded) conformal algebra Cend $M_{\gamma}$. There exists a natural map

$$
\pi_{\gamma}: C_{e} \rightarrow \text { Cend } M_{\gamma}, \quad \pi_{\gamma}\left(\sum_{i, j} a_{i j}(T, x) E_{i j}\right)=\sum_{i, j: \alpha_{i}=\alpha_{j}=\gamma} a_{i j}(T, \sigma(\gamma) x) E_{i j}
$$

It follows from (18) that $\pi_{\gamma}$ is a homomorphism of conformal algebras. Moreover, if $\mu_{\gamma}: M_{\gamma} \rightarrow M_{\gamma}$ is the map defined by $h(T) \otimes e \mapsto h(\sigma(\gamma) T) \otimes e$ then

$$
\mu_{\gamma}^{-1}\left(\pi_{\gamma}(a)\right)(\lambda) \mu_{\gamma}=\left.a(\lambda)\right|_{M_{\gamma}}
$$

for all $a \in C_{e}$. Therefore, irreducibility of $C$ implies $\pi_{\gamma}\left(C_{e}\right)$ is a finite irreducible subalgebra of Cend $M_{\gamma}$. By [16], the latter coincides with Cur End $\mathbb{k}^{n_{\gamma}}$ if we make an appropriate choice of an $H^{0}$-basis in $M_{\gamma}$. 
The latter means (see, e.g., [17]) that there exists an invertible matrix $Q_{\gamma} \in$ $M_{n_{\gamma}}(\mathbb{k}[x])$ such that

$$
Q_{\gamma}^{-1}(x) \pi_{\gamma}\left(C_{e}\right) Q_{\gamma}(x-T)=M_{n_{\gamma}}(\mathbb{k}[T]) .
$$

Let us choose a new $H^{0}$-basis in $M$ with transition matrix

$$
Q(T)=\bigoplus_{\gamma \in \Gamma \backslash \Gamma_{0}} Q_{\gamma}\left(\sigma(\gamma)^{-1} T\right) .
$$

In this basis, matrices of all elements from $C_{e}$ do not depend on $x$, i.e., $\pi_{\gamma}\left(C_{e}\right)=$ Cur End $\mathbb{k}^{n_{\gamma}}$.

By Proposition 3, $C_{e}$ is a semisimple finite conformal algebra. As shown in 5 (see also [17]), $C_{e}$ is isomorphic to the direct sum of current conformal algebras over matrix algebras, i.e.,

$$
C_{e}=I_{1} \oplus \cdots \oplus I_{p}, \quad I_{k} \simeq \text { Cur End } \mathbb{k}^{n_{k}} .
$$

Denote by $\pi_{k}$ the isomorphism Cur End $\mathbb{k}^{n_{k}} \rightarrow I_{k}$.

All conformal algebras Cur End $\mathbb{k}^{n_{k}}$ are simple, so we may split $\Gamma \backslash \Gamma_{0}$ into adjacent classes

$$
\Gamma_{k}=\left\{\gamma \in \Gamma \mid \pi_{\gamma}\left(I_{k}\right)=\operatorname{Cur} \operatorname{End} \mathbb{k}^{n_{\gamma}}\right\}, \quad k=1, \ldots, p .
$$

In particular, $n_{\gamma}=n_{k}$ for every $\gamma \in \Gamma_{k}$.

For every $k=1, \ldots, p$ and for every $\gamma \in \Gamma_{k}$ we may consider the automorphism $\theta_{\gamma}=\pi_{\gamma} \pi_{k}:$ Cur End $\mathbb{k}^{n_{k}} \rightarrow$ Cur End $\mathbb{k}^{n_{k}}$

Lemma 3. Let $\theta$ be an automorphism of the conformal algebra Cur End $\mathbb{k}^{n} \simeq$ $M_{n}(\mathbb{k}[T])$. Then $\theta(a)=Q^{-1} a Q, a \in \mathrm{Cur}$ End $\mathbb{k}^{n}$, for some invertible matrix $Q \in$ $M_{n}(\mathbb{k})$.

In particular, $\theta\left(E_{n}\right)=E_{n}$.

Proof. Let us extend $\theta$ to a map $\bar{\theta}: \operatorname{Cend}_{n} \rightarrow \operatorname{Cend}_{n}$, where $\operatorname{Cend}_{n}=\operatorname{Cend}(H \otimes$ $\left.\mathbb{k}^{n}\right) \simeq M_{n}(\mathbb{k}[T, x])$ by the following rule:

$$
\bar{\theta}\left(x^{k} a\right)=x^{k} \theta(a), \quad a \in \operatorname{Cur} \text { End } \mathbb{k}^{n}, k \geq 0 .
$$

It is easy to see that the map $\bar{\theta}$ obtained is an automorphism of the conformal algebra $\operatorname{Cend}_{n}$.

All automorphisms of $\operatorname{Cend}_{n}$ were described in [16, see also [17. There exist a scalar $\alpha \in \mathbb{k}$ and an invertible matrix $Q(t) \in M_{n}(\mathbb{k}[t])$ such that for a given $a=a(T, x) \in \operatorname{Cend}_{n}$ we have $\bar{\theta}(a(T, x))=Q^{-1}(x) a(T, x+\alpha) Q(x-T)$. Since $\bar{\theta}$ preserves Cur End $\mathbb{k}^{n}$ whose elements do not depend of $x$, the components of $Q$ do not depend on $x$ as well.

Therefore, for every $\gamma \in \Gamma_{k}, k=1, \ldots, p, \theta\left(E_{n_{k}}\right)=\pi_{\gamma}\left(\varepsilon_{k}\right)=E_{n_{k}}$, where $\varepsilon_{k}=\pi_{k}\left(E_{n_{k}}\right) \in I_{k}$. Since $\pi_{\gamma}$ is given by (19), $\varepsilon_{k}=E_{N_{k}}$, where $N_{k}=\left|\Gamma_{k}\right| n_{k}$. Hence, $C_{e}$ contains the identity matrix $E_{N}=\varepsilon_{1}+\cdots+\varepsilon_{p}$.

It remains to describe finite graded irreducible subalgebras $C \subseteq \operatorname{Cend}_{N}^{\Gamma}$ such that $\pi_{\gamma}\left(C_{e}\right)=$ Cur End $\mathbb{k}^{n_{\gamma}}$ for every $\gamma \in \Gamma \backslash \Gamma_{0}$ and $E=E_{N} \in C_{e}$.

Suppose $a=a(T, x)$ is a nonzero element of $C_{\alpha}$ for some $\alpha \in \Gamma$. Then

$$
a=\sum_{s \geq 0}\left(\sigma\left(\alpha^{-1}\right) T+\varphi\left(\alpha, \alpha^{-1}\right)\right) a^{s}(x), \quad a^{s}(x)=\sum_{i, j: \gamma_{i} \gamma_{j}^{-1}=\alpha} a_{i j}^{s}(x) E_{i j},
$$

where $a_{i j}^{s}(x) \in \mathbb{k}[x]$. 
Since $a_{(\lambda)} E \in C_{\alpha}$ for every $\lambda \in \mathbb{A}^{1}$, we may conclude $a^{s}(x) \in C_{\alpha}$ for all $s \geq 0$. Therefore, $C=H^{0} \otimes A$, where $A$ is a graded subspace of $M_{N}(\mathbb{k}[x])$ with respect to the same natural grading.

Assume $a=a^{0}(x)$, and choose $i, j \in\{1, \ldots, N\}$ such that $a_{i j}(x)=a_{i j}^{0} \neq 0$. Denote $\gamma=\gamma_{j}$, then $\gamma_{i}=\alpha \gamma$.

Since both $M_{\gamma}, M_{\alpha \gamma} \neq 0$, there exists $0 \neq b \in C_{\alpha^{-1}}$. We may assume $b=b(x)$, and choose $k, l \in\{1, \ldots, N\}$ such that $\gamma_{l}=\gamma, \gamma_{k}=\alpha \gamma, b_{l k}(x) \neq 0$.

For every $k \in\{1, \ldots, N\}$ such that $\gamma_{k}=\alpha \gamma$ there exists $\varepsilon_{k i} \in C_{e}$ such that $\pi_{\alpha \gamma}\left(\varepsilon_{k i}\right)=E_{k i} \in \operatorname{Cur} M_{n_{\alpha \gamma}}(\mathbb{k})$ (assuming the numeration of rows and columns in this "small" matrix is the same as in the initial $(N \times N)$-matrix $a)$.

Then $c=b_{(0)}\left(\varepsilon_{k i(0)} a\right) \in C_{e}$, but (7) implies $c(0) e_{j}=b(0) \varepsilon_{k i}(0) a(\lambda) e_{j}$. The latter is equal to $b_{l k}(T) a_{i j}(T+\varphi(\alpha, \gamma)) e_{l} \in M_{\gamma}$, i.e., if either of $a_{i j}$ or $b_{k l}$ depend on $x$ then some nonzero coefficients of $c \in C_{e}$ also depend on $x$. This is impossible since $C_{e}$ is a direct sum of current algebras, $\pi_{\gamma}\left(C_{e}\right)=\operatorname{Cur}_{\operatorname{End}} \mathbb{k}^{n_{\gamma}}=M_{n_{\gamma}}(\mathbb{k}[T])$.

Therefore, if we choose a basis of $M$ over $H^{0}$ as described above then all coefficients of all matrices in $A$ do not depend on $x$. Hence, $A$ is a graded subalgebra of $M_{N}(\mathbb{k}) \simeq$ End $V$. It is easy to see that the irreducibility of $C$ implies $A$ to be graded irreducible subalgebra of End $V$.

Corollary 1 implies

Corollary 3. A finite graded irreducible conformal subalgebra $C \subseteq \operatorname{Cend}_{N}^{\Gamma}$ is simple.

Corollary 4. A finite simple $(\Gamma, \sigma, \varphi)$-graded associative conformal algebra is isomorphic to the current graded conformal algebra Cur $A$ over a simple finite-dimensional graded associative algebra A.

Proof. By Proposition 2 and Theorem 2 we have $C \simeq$ Cur $A$ for a finite-dimensional graded associative algebra $A$. The graded algebra $A$ has to be simple since a nonzero proper graded ideal $I \triangleleft A$ produces similar ideal Cur $I$ in $C$.

Now, consider semisimple graded associative conformal algebras. We need a statement that can be proved in general settings.

Let $G^{0}$ be a connected linear algebraic group, $\Gamma$ be a group, and let $M$ be a finitely generated torsion-free $\Gamma$-graded $H^{0}$-module. As before, fix a homomorphism $\sigma: \Gamma \rightarrow$ Aut $G^{0}$ and a cocycle $\varphi \in Z^{2}\left(\Gamma, G^{0}, \sigma\right)$.

Lemma 4. Let $C$ be a semisimple $(\Gamma, \sigma, \varphi)$-graded associative conformal algebra over $G^{0}$, and let $M$ be a faithful graded conformal $C$-module which is torsion-free and finitely generated as an $H^{0}$-module. Then the set of all nonzero faithful graded $C$-submodules of $M$ contains a minimal element.

Proof. Suppose

$$
M=M_{0} \supseteq M_{1} \supseteq M_{2} \supseteq \ldots
$$

is a descending chain of faithful graded $C$-submodules. Since $M$ is Noetherian, all $M_{k}$ are finitely generated over $H^{0}$.

Consider the field of fractions $Q\left(H^{0}\right)$ and the linear space $Q(M)=Q\left(H^{0}\right) \otimes_{H}$ $M$. Since $M$ is torsion-free, $M$ is a $\mathbb{k}$-subspace of $Q(M)$ and all $M_{k}$ span finitedimensional $Q\left(H^{0}\right)$-subspaces of $Q(M)$. Therefore, there exists $N \geq 0$ such that for every $n>N$ there exists $0 \neq h \in H^{0}$ such that $h M_{N} \subseteq M_{n}$. 
By Lemma 1. $\left(C_{(\lambda)} M_{N}\right) \subseteq M_{n}$ for all $\lambda \in G^{0}$. The $H^{0}$-submodule $C M_{N}=$ $H^{0}\left\{\left(a_{(\lambda)} u\right) \mid a \in C, u \in M_{N}, \lambda \in G^{0}\right\}$ is a nonzero faithful graded conformal $C$-submodule of $M$ which is a lower bound of all $M_{k}$ in the initial chain. The Zorn Lemma implies the claim.

Theorem 3. If every finite irreducible $(\Gamma, \sigma, \varphi)$-graded subalgebra of $\mathrm{Cend}^{\Gamma} M$ is simple then every finite semisimple $(\Gamma, \sigma, \varphi)$-graded subalgebra of $\operatorname{Cend}^{\Gamma} M$ is a direct sum of simple ones.

Proof. Suppose $C \subseteq \operatorname{Cend}^{\Gamma} M$ is a semisimple $(\Gamma, \sigma, \varphi)$-graded subalgebra. It follows from Lemma 4 that there exists a minimal faithful graded conformal $C$ submodule $M_{0} \subseteq M$, so $C \subseteq \operatorname{Cend}^{\Gamma} M_{0}$.

Let $U$ be a maximal graded conformal $C$-submodule of $M_{0}$. If $U=0$ then $M_{0}$ is irreducible, so $C$ is simple. If $U \neq 0$ then $I=\{a \in C \mid a U=0\}$ is a nonzero graded ideal of $C$. Moreover, if $U^{\prime}$ stands for $\left\{v \in M_{0} \mid I v=0\right\} \subset M_{0}$ then $U=U^{\prime}$ from the maximality of $U$.

Denote $J=\{a \in C \mid I a=0\}$. This is a graded ideal in $C$, and $I \cap J=0$. Note that $J M \subseteq U^{\prime}=U$. Conversely, if $a M_{0} \subseteq U$ then $I a M_{0} \subseteq I U=0$, so $M / U$ is a faithful graded conformal $C / J$-module. This module is irreducible by the choice of $U$.

Hence, by the assumption of the Theorem, $C / J$ is simple, so $I \simeq C / J$, and $C=I \oplus J$. It is obvious that $J$ is semisimple, and we may apply the same procedure to $J$. Noetherianity of $C$ implies the process to stop on a finite number of steps, so $C=I_{1} \oplus \cdots \oplus I_{p}$, where $I_{k}$ are simple finite graded conformal algebras described by Corollary 4

Corollary 5. A finite semisimple $(\Gamma, \sigma, \varphi)$-graded associative conformal algebra over the affine line is a direct sum of simple ones.

Proof. It is enough to note that a finite semisimple graded conformal algebra $C$ embeds into $\operatorname{Cend}^{\Gamma} C$, and then apply Corollary 3 ,

\section{ACKNOWLEDGEMENTS}

The work is supported by the Russian Foundation for Basic Research (project 0901-00157) and the Federal Target Grant "Scientific and educational staff of innovation Russia" for 2009-2013 (contracts 02.740.11.5191, 02.740.11.0429, 14.740.11.0346). The author is grateful to Valerĭ Churkin for helpful discussions.

\section{REFERENCES}

[1] Kac, V.G.: Vertex algebras for beginners, Second Ed. University Lecture Series, vol. 10, AMS, Providence, RI (1996)

[2] Belavin, A.A., Polyakov, A.M., Zamolodchikov, A.B.: Infinite conformal symmetry in twodimensional quantum field theory. Nucl. Phys. 241, 333-380 (1984).

[3] Beilinson, A.A., Drinfeld, V.G.: Chiral algebras. Amer. Math. Soc. Colloquium Publications, 51, AMS, Providence, RI (2004).

[4] Bakalov, B., D'Andrea, A., Kac, V.G.: Theory of finite pseudoalgebras. Adv. Math. 162, 1, 1-140 (2001).

[5] D'Andrea, A., Kac, V.G., Structure theory of finite conformal algebras. Sel. Math., New Ser. 4, 377-418 (1998).

[6] Kolesnikov, P.: On irreducible algebras of conformal endomorphisms over a linear algebraic group. J. Math. Sci. 161, no. 1, 41-56 (2009). 
[7] Frenkel, I.B., Lepowsky, J., Meurman, A.: Vertex operator algebras and the Monster. Pure and Applied Math. 134, Academic Press, Boston (1998).

[8] Roitman, M.: On free conformal and vertex algebras. J. Algebra 217, no. 2, 496-527 (1999).

[9] Ginzburg, V., Kapranov, M.: Kozul duality for operads. Duke Math. J. 76, no. 1, 203-272 (1994).

[10] Kolesnikov, P.: Identities of conformal algebras and pseudoalgebras. Comm. Algebra 34, no. 6, 1965-1979 (2006).

[11] Golenishcheva-Kutuzova, M.I., Kac, V.G.: Г-conformal algebras. J. Math. Phys. 39, no. 4, 2290-2305 (1998).

[12] Brown, K.S.: Cohomology of groups. Graduate Texts in Mathematics, 87. Springer-Verlag, New York-Berlin (1982).

[13] Berezin, F.A., Introduction to superanalysis. D. Reidel Publishing Company, Dordrecht (1987).

[14] Bahturin, Yu.A., Sehgal, S.K., Zaicev, M.V.: Group gradings on associative algebras. Journal of Algebra 241, no. 2, 677-698 (2001).

[15] Nastasescu, C., van Oystaeyen, F.: Graded ring theory. North-Holland Mathematical Library, 28. North-Holland Publishing Co., Amsterdam-New York (1982).

[16] Boyallian, C., Kac, V.G., Liberati, J.-I.: On the classification of subalgebras of Cend ${ }_{N}$ and $\mathrm{gc}_{N}$. J. Algebra 260, no. 1, 32-63 (2003).

[17] Kolesnikov, P.: Associative conformal algebras with finite faithful representation. Adv. Math. 202, no. 2, 602-637 (2006).

Sobolev Institute of Mathematics, Novosibirsk, Russia

E-mail address: pavelsk@math.nsc.ru 